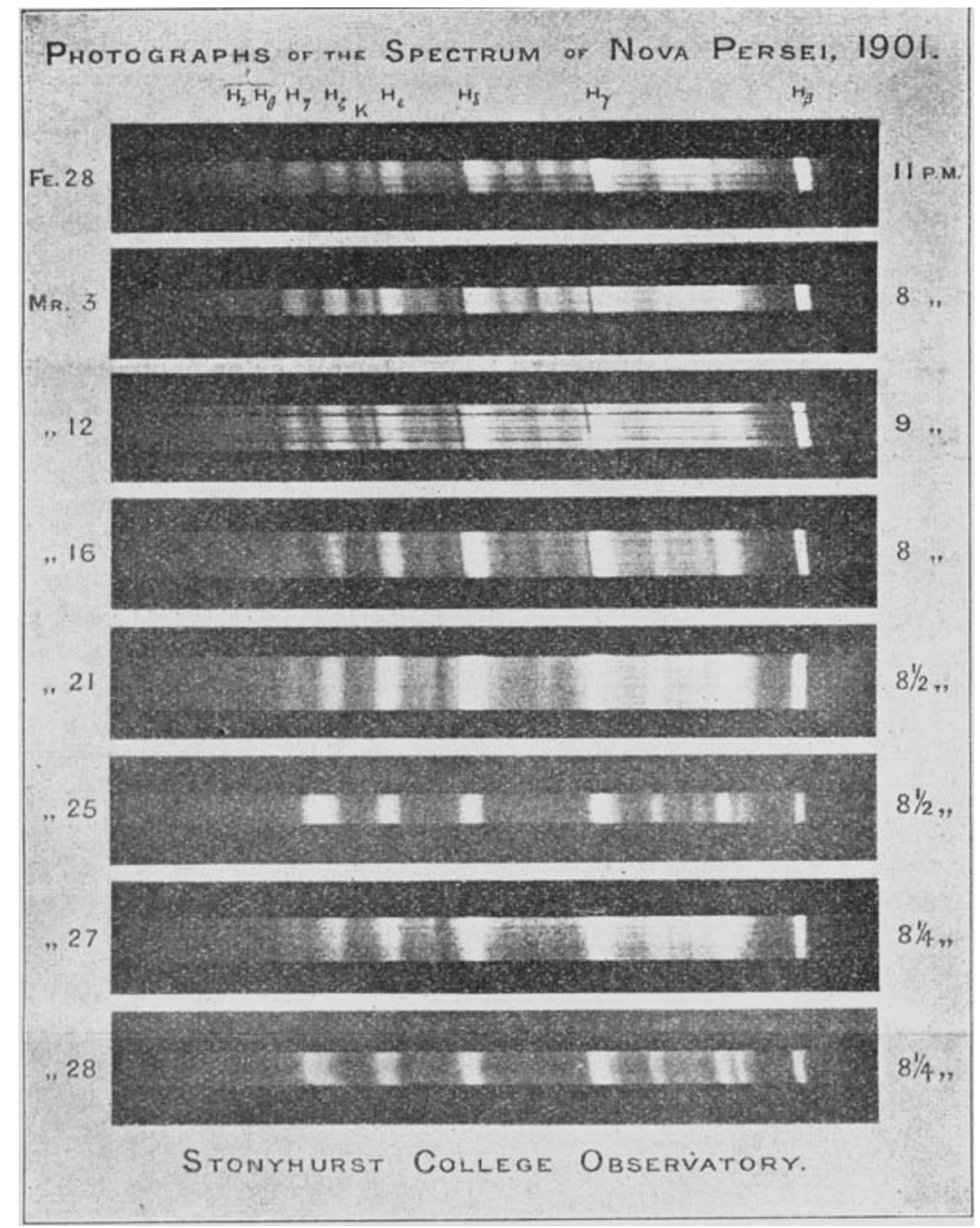

On March 3 1 and April 3, supposed minima, the sky was overcast. On April 4 a good photograph showed the same spectrum as on the $27^{\text {th }}$ March except that $H \zeta$ was marked by a strong line of maximum brightness considerably

Stonyhurst College Observatory, I90 I April. to the violet side of its wave-length centre. The star was: brighter; its continuous spectrum was stronger; and the $H \zeta$ extension absent.

\title{
Les fluctuations d'éclat de la Nova (3.1901) Persei.
}

Conformément à ce que j'ai annoncé dans mon télégramme du I 3 courant,*) la Nova Persei a continué à montrer des fluctuations d'éclat avec tous les caractères de périodicité. Dans le diagramme suivant $j$ 'ai tracé d'une façon approchée et provisoire la courbe moyenne des observations. Toutes celles-ci sont faites par

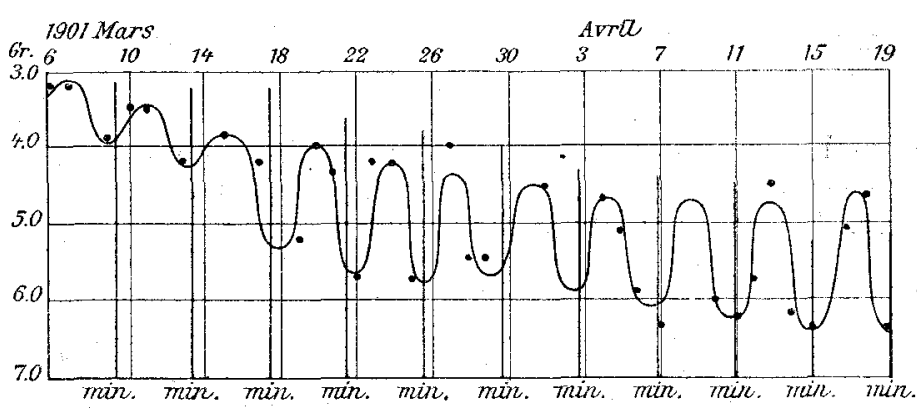

moi, exceptée celle du 26 mars qui appartient a M. Moye, observation très importante dans ce cas puisque elle correspond à un minimum. J'adopte seule. ment mes observations parce que la plupart de celles que j'ai pu voir, faites par des observateurs différents, sont à peine comparables entre elles.

*) Das Telegramm lautete : »Fluctuations éclat Nova Persei très probablement périodiques, période approchée $4^{j} \mathbf{2}^{\mathbf{h}}$. Comas Solá. $K^{r}$. 
Mes observations, qui commencent le 6 mars, ont été faites par la comparaison de la Nova avec les étoiles $\delta, v, x$, 1 et 30 Persei, me servant de la méthode des degrés. J'ai adopté les grandeurs suivantes de ces étoiles:

$$
\delta=3.5 ; \nu=4.1 ; x=4.4 ; 1=5.5 ; 30=6.4 \text {. }
$$

Dans la table suivante, les observations du 13 mars et 6 avril sont de faible poids à cause de brouillards.

\begin{tabular}{|c|c|c|c|c|c|c|c|}
\hline Mars & 6 & 3.2 & Mars 2 & 22 & $5 \cdot 7$ & Avril Io & 6.0 \\
\hline & 7 & 3.2 & & 3 & 4.2 & I I & 6.2 \\
\hline & 9 & 3.8 & & 4 & 42 & 12 & $5 \cdot 7$ \\
\hline & 10 & 3.5 & & 7 & 40 & I 3 & 4.5 \\
\hline & I I & $3 \cdot 5$ & & 28 & $5 \cdot 5$ & I 4 & 6.2 \\
\hline & 13 & $4.2 \pm$ & & 29 & $5 \cdot 5$ & I 5 & 6.4 \\
\hline & 15 & 3.8 & Avril & $\mathbf{I}$ & 4.5 & 17 & 5.1 \\
\hline & 17 & 4.2 & & 4 & 4.7 & 18 & $4.7^{*}$ \\
\hline & 19 & $5 \cdot 4$ & & 5 & 5.1 & I 9 & 6.2 \\
\hline & 20 & 4.0 & & 6 & $5.9 \pm$ & & \\
\hline & 21 & $4 \cdot 3$ & & 7 & $6 \cdot 3$ & & \\
\hline
\end{tabular}

Barcelone, Observatorio privado, le I 9 avril Igor.
A part la première partie des observations générales de la nouvelle étoile (du 2 I février au 6 mars) qui ne présentent visiblement aucune loi de périodicité, soit par les observations peu comparables, soit par les perturbations d'ordre physique qui peuvent avoir eu lieu dans l'étoile pendant l'époque de sa plus grande activité, il est de toute évidence, au moins jusqu'au présent, que les fluctuations d'éclat suivent une allure périodique, s'effectuant en 4 jours et 2 heures environ. On remarque aussi que l'amplitude des oscillations est plus grande à la fin qu'au commencement de mes observations.

Je crois qu'on pourrait expliquer très bien ces oscillations de lumière en supposant une région de l'atmosphère de l'étoile nouvelle plus brillante que le reste et de plus un mouvement de rotation à l'étoile qui produirait des éclipses de la plage brillante atmosphérique. La période de rotation de l'étoile sérait donc de 4 jours et 2 heures environ. Les erreurs d'observation et peut-être les alterations physiques de l'étoile pourraient expliquer quelques anomalies qu'on remarque dans la position de plusieurs points du diagramme.

\section{Beobachtungen des Cometen 1901 a.}

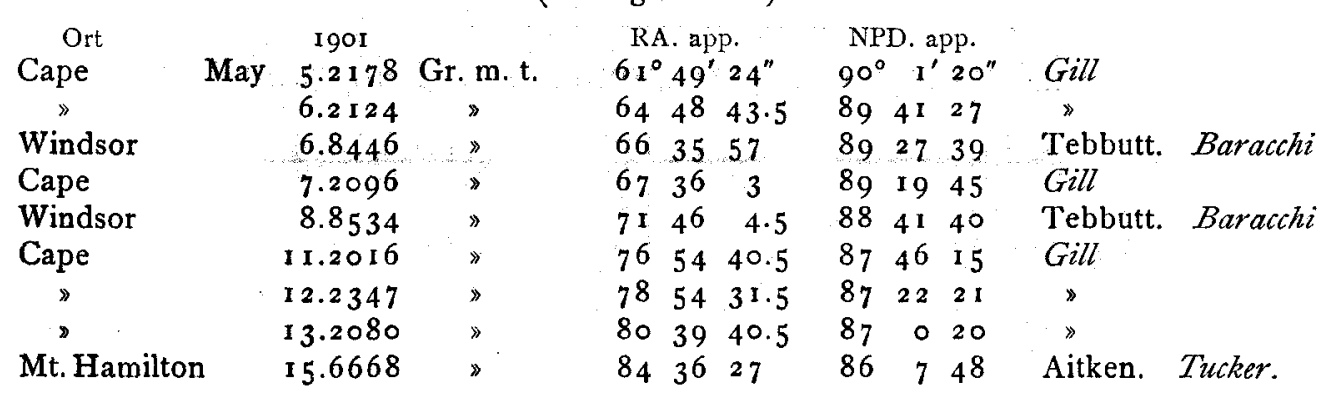

( $\mathrm{Telegramme}$.

\section{Elemente des Cometen 1901 a.}

Sir David Gill theilt telegraphisch die nebenstehenden Elemente mit, welche von Innes aus den Beobachtungen Mai 3, 5 und 7 berechnet worden sind.

\section{Comas Solá.}

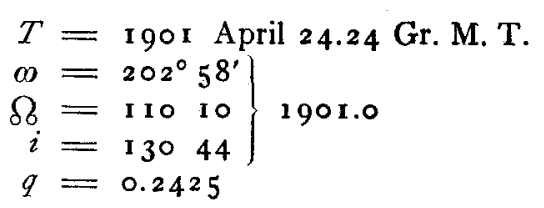

\section{Elementi ed effemeride del pianeta (416) Vaticana per la prossima opposizione.}

L' opposizione del $1900 \mathrm{mi}$ ha permesso di correggere $/$ stesso. Nei seguenti elementi, sui quali e basata l' effemeride leggermente gli elementi del pianeta, adottando un' orbita che soggiungo, ho tenuto conto delle perturbazioni da parte che rappresenta bene tutte le osservazioni che si hanno dello. di Giove e di Saturno.

Epoca ed osculazione 1901 Maggio 29.5 t. m. Berlino.

$$
\begin{aligned}
& M=6^{\circ} 13^{\circ} \text { 0.1 } 8 \\
& \omega=1953^{6} \quad 1.8 \mathrm{I} \\
& \delta=5^{8} 3^{2} 3^{6.41} \text { I } \begin{array}{r}
\text { Eclitt. } \\
1900.0
\end{array} \\
& \varphi=12^{\circ} 36^{\prime} 33^{\prime \prime} 5^{2} \\
& \mu=760.75913 \\
& \log a=0.445^{8} 396
\end{aligned}
$$

